\title{
Development and targeted use of nilotinib in chronic myeloid leukemia
}

\author{
Carmen Fava \\ Hagop Kantarjian \\ Jorge Cortes \\ Elias Jabbour \\ Department of Leukemia, \\ The University of Texas \\ MD Anderson Cancer Center, \\ Houston, Texas, USA
}

Correspondence: Elias Jabbour

Department of Leukemia, Unit 428, The University of Texas MD Anderson Cancer Center, 1515 Holcombe Blvd, Houston, TX 77030, USA

Tel + I 7137927305

Fax +I 7I3 7944997

Email ejabbour@mdanderson.org

\begin{abstract}
The development of imatinib has resulted in sustained hematologic and cytogenetic remissions in all phases of chronic myeloid leukemia (CML). Despite the high efficacy, relapses have been observed and are much more prevalent in patients with advanced disease. The most common mechanism of acquired resistance has been traced to Bcr-Abl kinase domain mutations. Several strategies have been developed to overcome the problem of imatinib resistance, including imatinib dose escalation, novel targeted agents and combination treatments. A second generation of tyrosine kinase inhibitors was developed, which displays increased potency towards Bcr-Abl and is able to target the majority of CML mutant clones. Nilotinib (Tasigna ${ }^{\circledR}$, AMN107, Novartis) is a close analog of imatinib with approximately 20-fold higher potency for BCR-ABL kinase inhibition. Preclinical and clinical investigations demonstrate that nilotinib effectively overcomes imatinib resistance, and has induced high rates of hematologic and cytogenetic responses in CML post imatinib failure, with a good tolerance. Nilotinib has been approved for CML patients in chronic and accelerated phases, post imatinib failure.
\end{abstract}

Keywords: nilotinib, imatinib-resistance, imatinib-intolerance, CML

\section{Ph-positive chronic myeloid leukemia}

Chronic myeloid leukaemia (CML) is a rare disease. Although its incidence is low, the prevalence of CML is increasing. In the US, the annual incidence of CML is 1.5 cases $/ 100,000$ adults, accounting for $15 \%$ of all of the forms of diagnosed leukemia (Ries et al 2004).

CML is one of the most extensively studied and, arguably, best understood neoplasms. CML is the first malignant disease for which a direct gene link has been found and there has been much recent progress in the understanding of the biology of the disease (Faderl et al 1999; Barnes and Melo 2002; Litzow 2006; Quintas-Cardama and Cortes 2006). The cytogenetic hallmark of CML is the Philadelphia chromosome $(\mathrm{Ph})$, a reciprocal translocation between chromosomes 9 and $22(\mathrm{t}(9 ; 22)(\mathrm{q} 34 ; \mathrm{q} 11))$. This translocation is present in 95\% of patients with CML (Rowley 1973).

The conjugation of the breakpoint cluster region gene on chromosome 22 and the Abelson $(\mathrm{Abl})$ kinase gene on chromosome 9 is a product of unfaithful repair of 2 DNA double-strand breaks in genes encoding BCR and ABL that creates the chimeric BCR-ABL oncogene, which codes for a deregulated tyrosine kinase (Koptyra et al 2006). The $210 \mathrm{kDa} B c r-A b l$ protein is expressed in CML patients, whereas a $190 \mathrm{kDa}$ Bcr-Abl protein, resulting from an alternative breakpoint in the $B C R$ gene, is expressed in $\mathrm{Ph}+$ acute lymphoblastic leukaemia (ALL) patients (Chan et al 1987).

Bcr-Abl displays transforming activity owing to its constitutive kinase activity, which results in multiple signal transduction pathways, including Ras/Raf/ mitogen-activated protein kinase (MAPK), phosphatidylinositol 3 kinase, STAT5/Janus kinase, and Myc, leading to uncontrolled cell proliferation and reduced apoptosis and 
resulting in the malignant expansion of pluripotent stem cells in bone marrow (Bhatia et al 2003; Hu et al 2004).

The Bcr-Abl kinase itself enhances genomic instability leading to accumulation of secondary genetic errors, which may be responsible for resistance to small molecule drugs, such as imatinib, and transformation to advanced phases (Koptyra et al 2006).

CML normally progresses through 3 clinically recognized phases. Approximately $90 \%$ of patients are diagnosed during the typically indolent chronic phase (CP), which is followed by an accelerated phase (AP) and a terminal blastic phase (BP).

The criteria for passage into CML-AP and CML-BP are defined hematologically on the basis of blast cell and progenitor cell counts. CML-AP persists for several months before the transition into CML-BP, typically defined as $30 \%$ blasts in the peripheral blood or bone marrow. Median survival in CML-BP is 3 to 6 months. CML-BP can manifest as myeloid, lymphoid, or undifferentiated phenotypes. Although progression through all stages is most common, $20 \%$ to $25 \%$ of patients progress directly from CP to BP. The time course for progression can also be extremely varied.

The mechanisms behind CML disease progression are not fully understood. As patients progress through the different phases, cytogenetic abnormalities may be detected in addition to the $\mathrm{Ph}$ chromosome (termed clonal evolution). Besides the propensity of the Ph-positive clone to acquire additional genetic changes, the BCR-ABL gene itself may acquire mutations that allow an already genetically unstable phenotype to further changes. Furthermore mutations and deletions in other specific genes may also occur (eg, p53, p16/INK4a, and RB). Increasing evidence suggests that Src family kinases may be involved in CML disease progression through the induction of cytokine independence and apoptotic protection (Lionberger et al 2000; Donato et al 2003; Dai et al 2004).

One of the major differences between the CP and BP of CML is their differential responsiveness to antileukemia treatment including tyrosine kinase inhibitors. Whereas most patients in $\mathrm{CP}$ achieve sustained responses, responses in patients with BP are usually transient.

In selected patients, allogeneic stem cell transplantation (ASCT), can be curative (Goldman and Melo 2003). Many patients, however, are not candidates for ASCT because of advanced age, the extent and/or duration of disease, or lack of availability of well-matched donors. The curative ability of ASCT resides, in part, in a direct graft-versus-leukemia effect that has permitted reductions in the intensity of ablative chemotherapy and/or substitution of immunosuppressive agents, with consequent extension of this treatment to patients previously not considered candidates for ASCT. Graft-versus-host disease and opportunistic infections cause significant mortality and morbidities threatening survival after ASCT, particularly in the first 1 to 3 years after the transplant.

Because of the high associated mortality rates $(10 \%-20 \%$ within the first year post treatment) and serious adverse events, even in patients with favorable prognostic criteria (matched sibling donor, CP disease diagnosed within 1 year and $<40$ years of age), ASCT is considered a salvage approach after tyrosine kinase inhibitors therapy failure (Quintas-Cardama and Cortes 2006).

\section{Treatment with imatinib}

The introduction of imatinib represented a breakthrough for CML therapy and for the targeted approach to cancer therapy. Imatinib, a small-molecule tyrosine kinase ihibitor (TKI), was the first drug to be developed that targets Bcr-Abl (Deininger et al 2005). Imatinib binds to the inactive protein conformation, partially occluding its ATP-binding site (Schindler et al 2000). Thus imatinib prevents the switch to the active conformation of Bcr-Abl, thereby blocking signal transduction. Imatinib also inhibits other signaling proteins; for example, platelet-derived growth factor receptor and c-Kit (Buchdunger et al 2000). The efficacy of imatinib against Ph-positive CML was demonstrated in various in vitro and in vivo preclinical models (Deininger et al 2005). The clinical benefits of this drug were subsequently shown in numerous clinical trials.

The prospective International Randomized trial of Interferon plus cytarabine versus imatinib [STI-571] (IRIS) Study of 1106 patients with newly diagnosed CML in CP established the superiority of imatinib $400 \mathrm{mg}$ daily over interferon-alpha and low-dose cytarabine (O'Brien et al 2003). The complete hematologic response rates were $95 \%$ versus $55 \%$; the complete cytogenetic response rates were $76 \%$ versus $15 \%$; and the progression-free survival rates at 18 months were $97 \%$ versus 91\% ( $\mathrm{p}<0.001)$. The molecular response rates were also significantly better, with estimated major molecular response rates at 12 months of $40 \%$ versus $2 \%$ (O'Brien et al 2003).

A 5-year update of the IRIS study continued to show positive results (Druker et al 2006). Three hundred and eighty-two patients remained on imatinib front-line therapy. 
The cumulative complete hematologic response, major cytogenetic response, and complete cytogenetic response rates were $98 \%, 92 \%$, and $87 \%$, respectively. The estimated 5 -year event-free survival was $83 \%$; only $6.3 \%$ of patients progressed to accelerated and blastic phases. The overall annual progression rate has declined to $0.9 \%$ in the fifth year of therapy, compared with $1.5 \%, 4.8 \%$, and $7.5 \%$ in the previous 3 years, suggesting that disease progression may be diminished in the following years. The estimated 5-year survival rate was $89 \%$; excluding non-CML deaths, it was $95 \%$. The intensity of the cytogenetic response after 12 and 18 months of imatinib therapy has important implications for survival without transformation. The estimated 5-year survival rate in patients not achieving a major cytogenetic response at 12 months was significantly less (81\%) than those who achieved major cytogenetic response (complete $97 \%$, partial 93\%; p < 0.001). At 18 months of therapy, the estimated 5-year survival rate for patients not achieving a complete cytogenetic response was significantly less than those who achieved complete cytogenetic response (99\% versus $90 \%$; p $<0.001)$. There was a continuous improvement in the rate of molecular response: the rate of major molecular response improved from $44 \%$ at 1 year to $68 \%$ at 4 years of therapy (Goldman et al 2005). This study did not document a survival advantage for imatinib because of the crossover design. Studies comparing the survival of patients treated with imatinib with historical cohorts treated with interferon-alpha-based therapy demonstrated the anticipated survival advantage (Kantarjian et al 2003; Roy et al 2006) .

\section{Imatinib adverse events}

Adverse events (AEs) reported with imatinib are generally mild or moderate, and included superficial edema (60\%), nausea (50\%), muscle cramps (49\%), and rashes (according to the common terminology criteria rare version 3.0). Grade 3-4 events were uncommon except for neutropenia (17\%) and thrombocytopenia (9\%). Only 4\% of patients had to discontinue imatinib because of AEs (Druker et al 2006).

\section{Resistance to imatinib}

Despite the benefit of imatinib over prior treatments, some patients may develop resistance (Shah 2005), with a reported annual relapse rate of $1 \%$ to $4 \%$ in newly diagnosed patients in chronic phase with the incidence decreasing over time (Hochhaus and Hughes 2004). In fact, during the 4th and the 5 th year of follow-up on the IRIS trial, less than $1 \%$ of patients have lost response per year. Multiple mechanisms of resistance to imatinib, Bcr-Abl-dependent and Bcr-Ablindependent, have been identified; one of the best characterized is mutations in the Bcr-Abl kinase domain. Mutations have been detected in $30 \%$ to $50 \%$ of patients who develop imatinib resistance (Hochhaus et al 2002). Clinically relevant mutations disrupt critical contact points between imatinib and $\mathrm{Bcr}-\mathrm{Abl}$ or may favor the active conformation of Bcr-Abl, to which imatinib is unable to bind (Deininger et al 2005; Shah 2005). Numerous Bcr-Abl mutations have been identified. Not all mutations have the same biochemical and clinical properties: some Bcr-Abl mutations result in a highly resistant phenotype in vitro; others are still relatively sensitive to imatinib and resistance may potentially be overcome by a dose increase of imatinib (Corbin et al 2002; Hochhaus et al 2002; Shah et al 2002; von Bubnoff et al 2002; Azam et al 2003). The T315I mutation and some mutations affecting the so-called p-loop of Bcr-Abl confer a greater level of resistance to imatinib and to the novel tyrosine kinase inhibitors (Hughes et al 2006; Jabbour et al 2006).

Other mechanisms of imatinib resistance may include: increased expression of Bcr-Abl through genomic amplification (Nicolini et al 2006), overexpression of Lyn or other Src-family tyrosine kinases (Donato et al 2003; Dai et al 2004), or overexpression of drug efflux proteins such as P-glycoprotein, which may decrease the intracellular concentration of imatinib in leukemic cells (Thomas et al 2004). Interestingly, treatment responses correlate with plasma concentrations of imatinib, suggesting that insufficient plasma levels may contribute to imatinib resistance. ${ }^{34}$ In addition, clonal evolution may also be involved in imatinib resistance through inactivation of the tumor suppressor gene p53 (Wendel et al 2006). Strategies to overcome imatinib resistance are a logical progression for improving the prognosis of patients with CML.

\section{TKIs: The next generation}

New TKIs are available or in development that are more potent than imatinib for inhibiting Bcr-Abl and have a decreased potential for resistance. These include dasatinib (Sprycel ${ }^{\circledR}$; BMS-354825, Bristol-Myers-Squibb), an orally bioavailable dual Bcr-Abl and Src inhibitor, and nilotinib (Tasigna $^{\circledR}$; AMN-107, Novartis), a potent selective Bcr$\mathrm{Abl}$ inhibitor. Both nilotinib and dasatinib induced significant clinical responses. Dasatinib was approved for the treatment of patients with all phase CML or Ph+ ALL who are unable to tolerate or have not responded to other treatments, including imatinib. Nilotinib was approved 
for CML patients in chronic and accelerated phases, post imatinib failure. Here we review the recent available data on nilotinib.

\section{Nilotinib}

\section{Preclinical profile}

Nilotinib (formerly AMN107, Novartis Pharmaceuticals, Basel, Switzerland) is an oral TKI that was developed from its parent compound, imatinib, as a more potent inhibitor of Bcr-Abl (Golemovic et al 2005; Verstovsek et al 2005). Rational design of novel inhibitors exhibiting effectiveness against imatinib-resistant mutants of Bcr-Abl was carried out, based on the crystal structure of the imatinib-Abl complex. Crystallographic analysis has revealed that imatinib binds to and stabilizes an inactive, DFG (aspartate-phenylalanine-glycine)-out conformation of Abl in which the activation loop is in a "closed" substratemimicking position and there is extensive distortion of the ATP binding loop.

The high topological congruency between the anilinepyrimidine substructure and the surface of the distorted ATP-binding pocket suggest that making changes within this region of imatinib might not be very productive. However, the methylpiperazinyl group of imatinib lies along a surfaceexposed pocket of the Abl kinase and is potentially more amenable to modification. It was hypothesized that the potency and selectivity of imatinib could be improved by maintaining binding to the inactive conformation of the Abl kinase domain, but incorporating alternative binding groups to the N-methylpiperazine moiety, while preserving an amide pharmacophore to retain $\mathrm{H}$-bond interactions to Glu286 and Asp381. Like imatinib, nilotinib binds to the inactive conformation of the Abl tyrosine kinase, with p-loop folding over the ATP-binding site, and the activation-loop (involving the pyridyl-N and the backbone-NH of Met318, the anilino-NH and the side-chain hydroxyl of Thr315, the amido-NH and side-chain carboxylate of Glu286, as well as the amido- $\mathrm{C}=\mathrm{O}$ and backbone-NH of Asp381) blocking the substrate binding site, to disrupt the ATP-phosphate-binding site, and inhibit the catalytic activity of the enzyme (Manley et al 2005).

This better fit between nilotinib and the kinase binding site, as well as differences in interactions with specific amino acid residues, led to the development of a high-affinity ATP-competitive inhibitor, 10 to 30 times more potent than imatinib, which decreases proliferation and viability of wild-type Bcr-Abl- and imatinib-resistant Bcr-Abl mutantexpressing cells in vitro by selectively inhibiting Bcr-Abl autophosphorylation (Schindler et al 2000; Nagar et al 2002; Manley et al 2005; Weisberg et al 2005). Nilotinib inhibits the kinase activity of most Bcr-Abl mutants, except for T315I (also resistant to imatinib and dasatinib) (O'Hare et al 2005). Like imatinib, nilotinib does not inhibit Src kinase and does not bind to the inactive conformation of Bcr-Abl.

Nilotinib was tested alongside imatinib and dasatinib in $16 \mathrm{Bcl}-\mathrm{Abl}$ mutant cell lines representing $>90 \%$ of clinical isolates. In recombinant kinase assays, $\mathrm{IC}_{50}$ values for inhibition of substrate phosphorylation by wildtype Abl were 280, 15 and $0.6 \mathrm{nM}$ for imatinib, nilotinib and dasatinib, respectively. A reduced susceptibility of $>10$-fold compared with wild type was seen in 7 mutants with imatinib (3 of which were completely insensitive) and 3 with nilotinib ( $\mathrm{IC}_{50}$ for $13 / 16$ mutants $<160 \mathrm{nM}$ ). The activity of dasatinib against 15 mutants remained between 0.5 -fold and 1.5 -fold of that against wildtype (all $\mathrm{IC}_{50}$ values $<1.8 \mathrm{nM}$ ). Inhibition of the proliferation of $\mathrm{BaF} 3$ cells expressing the mutant kinases followed a similar pattern for the three agents. $\mathrm{IC}_{50}$ values were higher and more variable for imatinib (480-3475 nM; 3 mutants completely resistant) and nilotinib (15-450 nM) than for dasatinib (0.8-7.4 nM).

Nilotinib inhibits Abl-catalyzed peptide substrate phosphorylation with a 20 -fold higher potency than imatinib (IC50 [concentration of inhibitor resulting in a $50 \%$ reduction in cell viability]: 15 versus $280 \mathrm{nmol} / \mathrm{L}$ ). Corresponding experiments with mutant Abl kinase domains revealed that a 20 -fold improved potency of nilotinib compared with imatinib is also seen with the imatinib-resistant mutants. A model of nilotinib in complex with Abl kinase mutant M351T showed that the sensitivity of Bcr-Abl mutants to nilotinib segregates into 4 categories: high (IC50 < $70 \mathrm{nmol} / \mathrm{L}: \mathrm{M} 244 \mathrm{~V}, \mathrm{G} 250 \mathrm{E}$, Q252H, F311L, F317L, M351T, V379I, L387M, H396P, H396R), medium (IC50 < $200 \mathrm{nmol} / \mathrm{L}:$ Y253F, E255K, F359V), low (IC50 < $450 \mathrm{nmol} / \mathrm{L}: \mathrm{Y} 253 \mathrm{H}, \mathrm{E} 255 \mathrm{~V}$ ), and insensitive (IC50 > $2 \mu \mathrm{mol} / \mathrm{L}$ : T315I), with the level of nilotinib sensitivity at positions 253 and 255 dependent on the specific amino acid substitution (mutants Y253F and E255K fall in the medium classification, whereas $\mathrm{Y} 253 \mathrm{H}$ and E255V comprise the low category). The mutants, other than T315I, that were least responsive to nilotinib in all three cellular assays were $\mathrm{Y} 253 \mathrm{H}$ and E255V. The pattern of resistance to nilotinib and dasatinib differed; those with greatest resistance to nilotinib ( $\mathrm{Y} 253 \mathrm{H}$ and E255V) were susceptible to dasatinib. T315I remained resistant to all the three drugs.

In vivo experiments with CML xenografts in mice demonstrated that nilotinib treatment, compared with 
treatment with vehicle, improved survival of mice infused with either wild type Bcr-Abl or the imatinib-resistant Bcr-Abl mutant (E255V) (Golemovic et al 2005; O'Hare et al 2005).

Although nilotinib and imatinib exhibit great selectivity for Bcr-Abl, Kit and Pdgf-R, these agents bind these kinases with different affinities. The ranking of imatinib affinities is Pdgf-R $>$ Kit $>$ Bcr-Abl, whereas for nilotinib this is Bcr-Abl $>$ Pdgfr $>$ Kit. Nilotinib had no significant effect on the variability of proliferation of $\mathrm{Ba} / \mathrm{F} 3$ cells rendered factor-independent through expression of several other tyrosine kinases such as ErbB2, Flt3, Met, Ret, Igf- 1 or Npm-Alk at concentrations $\leq 3 \mu \mathrm{M}$ (Weisberg et al 2005).

Nilotinib was also shown to inhibit cell proliferation of the KBM5 line, which over expressed Bcr-Abl, with IC50 values of $480 \mathrm{nM}$ (Golemovic et al 2005), and to revert the expression of the pre-apoptotic protein BCL-2interacting mediator both in vivo and ex vivo (Aichberger et al 2005).

In K562, the IC50 of nilotinib was $30 \mathrm{nM}$ versus $600 \mathrm{nM}$ for IM, consistent with its reported 20-fold-higher potency. However, in primary CD34-CML cells, nilotinib and imatinib were equipotent for inhibition of Bcr-Abl activity. CD34-cells were still able to expand over 72 hours with $5 \mu \mathrm{M}$ of either drug, although there was a concentration-dependent restriction of amplification. As for imatinib, the most primitive cells persisted and accumulated over 72 hours with nilotinib and remained. Furthermore, nilotinib with imatinib led to further accumulation of this population, suggesting at least additive anti-proliferative effects. These results confirmed that, like imatinib, the predominant effect of nilotinib is antiproliferative rather than proapoptotic (Jorgensen et al 2007).

\section{Phase I clinical trial}

Nilotinib has shown promise in clinical trials with effectiveness in patients whose disease is caused by mutated forms of Bcr-Abl that do not respond to imatinib.

In a phase 1 dose-escalation study, nilotinib was evaluated in patients with imatinib-resistant CML in various phases or with Ph+ ALL (Kantarjian et al 2006).

Nilotinib was administered orally to 119 patients (17 in chronic phase disease, 56 in accelerated phase [10 with clonal evolution only], 24 in myeloid blast phase, and 9 in lymphoid blast phase, together with $13 \mathrm{Ph}+$ ALL patients), for up to 385 days. Significant clinical activity was identified in all CML phases. Among 12 patients with CML-CP, 11 achieved a complete hematologic remission (92\%) and 9 of 17 (53\%) a cytogenetic response (6 complete cytogenetic responses). Nilotinib also demonstrated activity against advanced disease: of the patients in AP, 38 of 51 (74\%) achieved a hematologic response, including 26 complete hematologic remission, 3 marrow responses and 9 returned to CML-CP; $31(55 \%)$ had a cytogenetic response including 7 partial cytogenetic response and 8 a complete cytogenetic response. In $\mathrm{BP}$, the hematologic response rate was $42 \%$ for patients in myeloid blast phase and 33\% for those in lymphoid blast phase: among 24 patients with myeloid CML-BP, 2 achieved complete hematologic remission and 6 returned to CML-CP; among 9 patients with lymphoid CML-BP, 1 achieved a marrow response and 2 returned to CML-CP. Seven patients with myeloid (29\%) and 2 with lymphoid (22\%) CML-BP achieved a cytogenetic response with nilotinib. One patient in each of the two groups achieved a complete cytogenetic response. Four patients with myeloid BP achieved a partial cytogenetic response (17\%). Responses were observed in most patients with Bcr-Abl mutants, according to expected in vitro data.

The dose levels initially evaluated were 50 to $1200 \mathrm{mg}$ once per day. Plasma level saturation was observed at more than $400 \mathrm{mg} /$ day. Doses of nilotinib of 400 or $600 \mathrm{mg}$ twice daily produced the best responses. Intra-patient dose escalation was permitted in patients with an adequate response and no dose-limiting toxicities.

The pharmacokinetics of nilotinib have been evaluated. The median time to peak serum concentrations of nilotinib was 3 hours and the mean peak concentration at steady state, reached by day 8 , in patients administered $400 \mathrm{mg}$ twice daily was 3.6 $\mu \mathrm{M}$. Nilotinib had an apparent half-life of 15 hours (Table 1). Peak concentration and area under the serum concentration-time curve at steady state increased with dose from 50 to $400 \mathrm{mg}$ and plateaued with doses greater than $400 \mathrm{mg}$. Non-linearity with higher nilotinib doses is thought to result from saturation of gastrointestinal absorption; dosing nilotinib at $400 \mathrm{mg}$ twice daily resulted in steady-state exposure greater than that observed with a single daily $800 \mathrm{mg}$ dose. Based on these data, twice daily dosing was selected for phase II studies of nilotinib.

The treatment was well tolerated. At the dose of $400 \mathrm{mg}$ twice daily, grade 3 or 4 nonhematologic adverse events included pruritus (3\%), increased total and conjugated (3\%) as well as unconjugated bilirubin levels (3\%), increased lipase levels (9\%), and increased levels of liver enzymes (3\%). Most frequent grade 3-4 adverse events were hematologic: the incidence of grade 3 or 4 thrombocytopenia 
Table I Phase 2 data for nilotinib second-line to imatinib failure

\begin{tabular}{|c|c|c|c|c|c|}
\hline \multirow[t]{4}{*}{ Disease } & \multirow[t]{4}{*}{$\mathbf{N}$} & \multicolumn{4}{|c|}{ \% Response } \\
\hline & & \multirow{2}{*}{\multicolumn{4}{|c|}{$\begin{array}{l}\text { Cytogenetic } \\
\text { response }\end{array}$}} \\
\hline & & & & & \\
\hline & & CHR & Major & Complete & Overall survival \\
\hline $\begin{array}{l}\text { CML } \\
\text { chronic }\end{array}$ & 321 & 77 & 57 & 41 & $91 \%$ (18 months) \\
\hline $\begin{array}{l}\text { CML } \\
\text { accelerated }\end{array}$ & 136 & 26 & 31 & 19 & $81 \%$ (12 months) \\
\hline CML blastic & 136 & II & 40 & 29 & $42 \%$ (I2 months) \\
\hline
\end{tabular}

Abbreviations: $\mathrm{CHR}$, complete hematologic response; $\mathrm{CML}$, chronic myeloid leukemia; ALL, acute lymphoid leukemia; Ph, Philadelphia chromosome.

was $25 \%$, and the incidences of grade 3 or 4 anemia and neutropenia were less than $10 \%$. Other adverse events were less frequent and were primarily grade 1-2. In this study, nilotinib was not associated with the edema frequently associated with imatinib.

Neutropenia and indirect self-limited reversible hyperbilirubinemia were observed mostly at the dose of $600 \mathrm{mg}$ twice daily. A nilotinib dose of $400 \mathrm{mg}$ twice daily was selected for the phase 2 studies based on efficacy and long-term side effects.

\section{Phase 2 clinical trials}

Consistent with phase 1 results, results from following phase 2 studies have demonstrated activity in all phases of disease. Thus, nilotinib has been approved in both the US and Europe for the treatment of patients with CML-CP and -AP who are resistant or intolerant to prior therapy, including imatinib. Three single-arm studies in patients with all phases of imatinib-resistant or imatinib-intolerant CML or Ph-positive ALL were recently updated (Ottmann et al 2007; Giles et al 2008; Kantarjian et al 2008; le Coutre et al 2008) (Table 2).

Intolerance was defined as any non-hematologic toxicity of grade 3 or higher severity, or of grade 2 or higher severity lasting more than 1 month or recurring more than 3 times despite dose reduction and maximal supportive care.

Table 2 Pharmacokinetics (Nilotinib 2008)

\begin{tabular}{ll}
\hline Route and formulation & Oral: 200 mg caps \\
\hline Absorption & $\mathrm{T}_{\max } 3$ hours \\
Plasma half-life & $15-17$ hours \\
Metabolism & By CYP 3A4, through oxidation \\
& and hydroxilation \\
Excretion & Mostly in feces \\
\hline
\end{tabular}

The definition of intolerance also included hematologic toxicity of grade 4 severity persisting for more than 7 days. Imatinib-intolerant patients who had previously demonstrated sensitivity to imatinib, as evidenced by a prior major cytogenetic response, were to be excluded from participation in the studies.

At the time of the report, 321 patients (71\% imatinibresistant; $21 \%$ imatinib-intolerant) with CML-CP treated with nilotinib were evaluable (Kantarjian et al 2008). Nilotinib was started at $400 \mathrm{mg}$ twice daily (bid), on an empty stomach, and escalated to $600 \mathrm{mg}$ bid for inadequate responses. Complete hematologic response was reported in 158 of 206 patients with active disease at the beginning (77\%). Overall, the major cytogenetic response rate was $57 \%$; $41 \%$ had a complete cytogenetic response. Major cytogenetic responses were observed in $125(55 \%)$ of the 227 imatinib-resistant patients and in $59(63 \%)$ of the 94 imatinib-intolerant patients. The median time to complete hematologic response and major cytogenetic response was 1.0 and 2.8 months, respectively. The majority of patients (84\%) maintained the major cytogenetic response for at least 18 months. At 18 months, the estimated overall survival rate was $91 \%$.

One hundred and thirty-six patients with CML-AP received at least 1 dose of nilotinib, with a median treatment duration of 210 days (le Coutre et al 2008). A confirmed hematologic response occurred in 69/129 patients (54\%); $26 \%$ had a complete hematologic response. Major cytogenetic responses occurred in 40/129 patients (31\%); $24 / 129$ patients (19\%) had complete cytogenetic responses. Thirty out of 104 patients (29\%) of imatinib-resistant patients and 10/25 imatinib-intolerant patients (40\%), had a major cytogenetic response. Time to first hematologic response and major cytogenetic response was 1 and 2.8 months, respectively. At 12 months, it was estimated that $57 \%$ of patients were without progression and the estimated overall survival rate was $81 \%$.

One hundred and thirty-six patients with CML-BP (myeloid, $\mathrm{n}=105$; lymphoid, $\mathrm{n}=31$ ) were treated with nilotinib $400 \mathrm{mg}$ twice daily (Giles et al 2008). The hematologic response rate was $21 \%$ and $11 \%$ achieved complete hematologic response. Major cytogenetic response was achieved in 55 patients (40\%), and 40 (29\%) had complete cytogenetic response. Overall survival at 12 months was $42 \%$. Treatment with nilotinib is ongoing in 13 patients (10\%). More than half of patients discontinued treatment due to disease progression.

In all three studies, nilotinib was well tolerated. The toxicity profile was consistent with the phase 1 study, 
with generally low and manageable rates of severe adverse events. The rate of grade 3-4 neutropenia was $30 \%$ in $\mathrm{CP}$, $39 \%$ in AP, and $67 \%$ in BP. The rate of grade 3-4 thrombocytopenia was $28 \%$ in $\mathrm{CP}, 41 \%$ in $\mathrm{AP}$, and $62 \%$ in $\mathrm{BP}$. Non-hematologic side effects were infrequent and were usually grade $1-2$. These included fatigue, pruritus, headache, muscle spasms, and gastrointestinal disturbances. Grade 3-4 non-hematologic adverse events were rare and included rash, headache, diarrhea, nausea, and fatigue. Transient self-limited elevations of indirect bilirubin were noted. One patient had sudden cardiac death. A low incidence of QT prolongation (QTcF $>500 \mathrm{msec},<1 \%$ ) was observed overall.

These reports suggest that nilotinib may provide a safe and effective treatment option for CML. There was minimal cross intolerance between imatinib and nilotinib (Jabbour et al 2008). Nilotinib was associated with less fluid retention, edema, cramps, and weight gain, or with pleural effusions.

\section{Front-line therapy}

As seen with imatinib resistance in CML, cancer cells have the capacity to mutate and acquire properties that enable them to develop resistance to treatment. As a significant proportion of patients are/become resistant to imatinib, earlier use of nilotinib (ie, as a front-line therapy) in all phases of CML may be beneficial in 2 potential ways: by promoting an early response, thereby potentially improving prognosis; and/or by avoiding the development of treatment resistance. The clinical challenge in this setting would be to accurately identify patients who are likely to fail imatinib treatment by using clinical risk factors and genetic testing as predictive factors.

A phase 2 study in patients with newly diagnosed CML-CP showed that nilotinib $400 \mathrm{mg}$ twice daily induces a complete cytogenetic response in nearly all patients as early as 3 months after the start of therapy with a favorable toxicity profile. Thirty-five patients have been treated for a median of 6.5 months. Complete cytogenetic responses were achieved, respectively, by $96 \%$ and by $100 \%$ of patients at 3-and 6-month evaluations. The rate of complete cytogenetic response at 3, 6 and 12 months compares favorably to those observed in historical controls treated with imatinib $400 \mathrm{mg}$ or $800 \mathrm{mg}$ daily: at 12 months $100 \%$ of patients were still in response. Major molecular response was observed in $13 \%$ at 3 months, $45 \%$ at 6 months and $45 \%$ at 12 months. Nilotinib showed generally low and manageable rates of grades 3-4 adverse events (Cortes et al 2008).

\section{Potential resistance to second generation TKIs}

As clinical experience with second generation TKIs grows, it is becoming clear that patients can also relapse on treatment after an initial response, particularly in the setting of advanced-phase CML. Although over expression of Bcr-Abl is a possible resistance mechanism for nilotinib (Mahon et al 2004), resistance is more likely to arise through the emergence of clones expressing nilotinib-resistant mutant forms of Bcr-Abl. With the increased use of these newer TKIs, it has been suggested that the spectrum of kinase domain mutations may change. As these compounds enter the clinical arena, it would be useful to predict the profile of mutations that confer drug resistance because this would allow for a rational approach to the design of combination strategies. Experimental models of in vitro drug sensitivity exposing Bcr-Abl-expressing cells to nilotinib or dasatinib under conditions that favor the development of mutations have shown that although their mutagenic potential is lower than that of imatinib, mutations may still emerge (Table 3).

Table 3 In vitro mutagenesis for resistance to nilotinib

\begin{tabular}{|c|c|c|}
\hline Von Bubnoff et $\mathrm{al}^{53}$ & Bradeen et $\mathrm{al}^{52}$ & Ray et $\mathrm{al}^{54}$ \\
\hline & & K247N \\
\hline & L248V & L248V \\
\hline & $\mathrm{G} 250 \mathrm{E}$ & \\
\hline \multirow[t]{2}{*}{ Q252H } & & $\mathrm{Q} 252 \mathrm{H}$ \\
\hline & & Y253C \\
\hline $\mathrm{Y} 253 \mathrm{H}$ & $\mathrm{Y} 253 \mathrm{H}$ & $\mathrm{Y} 253 \mathrm{H}$ \\
\hline E255K & E255K & E255K \\
\hline \multirow[t]{8}{*}{ E255V } & E255V & \\
\hline & & L273F \\
\hline & & $\mathrm{E} 282 \mathrm{~K}$ \\
\hline & & $\mathrm{K} 285 \mathrm{~N}$ \\
\hline & & V289L \\
\hline & & E292K \\
\hline & E292V & \\
\hline & & N297T \\
\hline \multicolumn{3}{|l|}{ F3III } \\
\hline T3I5I & T3I5I & $\mathrm{T} 3|5|$ \\
\hline \multicolumn{3}{|l|}{ S349L } \\
\hline & $\mathrm{F} 359 \mathrm{C}$ & \\
\hline \multicolumn{3}{|l|}{ F359l } \\
\hline \multicolumn{3}{|l|}{ F359V } \\
\hline & & $\mathrm{H} 375 \mathrm{P}$ \\
\hline & L384M & \\
\hline & L387F & \\
\hline & & T406I \\
\hline & & W470L \\
\hline
\end{tabular}


In a cell-line-based mutagenesis study, the emergence of Bcr-Abl mutations resistant to imatinib, nilotinib and dasatinib were compared: 20 different mutations were identified with imatinib mesylate, 10 with nilotinib (including only 1 novel mutation, E292V) and 9 with dasatinib. In contrast to imatinib mesylate, mutations recovered in the presence of $50 \mathrm{nM}$ nilotinib were limited to L248V, G250E, Y253H, E255K (p-loop), T315I, F359C, L384M and L387F. One novel mutation (E292V) was seen in a single clone at $50 \mathrm{nM}$. At $500 \mathrm{nM}$, only Y253H, E255V and T315I were detected, in line with their reported level of resistance in cell proliferation assays. At concentrations of at least $2000 \mathrm{nM}$, only T315I was recovered. The fact that only 1 novel mutation occurred at a low concentration and the lack of novel, previously undescribed mutations at intermediate inhibitor concentrations $(500 \mathrm{nM})$ suggests that the structural modifications of nilotinib compared with imatinib do not generate clinically relevant novel vulnerable sites. Because the plasma trough concentrations at the maximum tolerated dose (MTD) of nilotinib (400 mg twice daily) are in the range of $2000 \mathrm{nM}$, the prediction is that only T315I will emerge in patients treated with the MTD. Interestingly, with combination of nilotinib plus other TKIs such as imatinib or dasatinib, maximal suppression of resistant clone outgrowth was achieved with lower concentrations compared with single agents (Bradeen et al 2006).

An alternative cell-based screening assay designed to predict such mutations has recently been applied to nilotinib (von Bubnoff et al 2006). Using this system, a reduced pattern of mutations was observed for nilotinib, having some overlap with that seen for imatinib: Q252H, Y253H, E255K(V), F311I, T315I, S349L and F359I(V), all of which, with the exception of the T315I mutant, were suppressed at clinically achievable concentrations of nilotinib.

Bcr-Abl point mutations conferring resistance to nilotinib have also been identified in a random mutagenesis study (Ray et al 2005). In this study, 11 novel mutations were detected (K247N, L248V, L273F, E282K, K285N, V289L, E292K, N297T, H375P, T406I and W430L), in addition to 5 (Q252H, Y253C (H), E255K and T315I), which have been previously observed in CML patients treated with imatinib. Although these studies do not consistently identify the same drugresistant Bcr-Abl point mutations for individual drugs, it is clear that all 3 compounds display different mutagenicity profiles.

The patterns of mutations occurring in patients with all phase CML treated sequentially with imatinib, and 1 or 2 second-generation TKIs after imatinib failure were investigated (Cortes et al 2007). Response rates were similar for patients with and without mutations, regardless of mutation site except for T315I. Acquisition of kinase domain mutations was the most frequently identified mechanism of resistance for patients who failed a second-generation TKI therapy. The codons involved were diverse, and most frequently included those also seen after imatinib failure. However, some mutations occurred more frequently after specific second TKIs, such as F317L after dasatinib, and certain p-loop mutations after nilotinib. Few novel mutations also emerged, such as V299L after dasatinib, and in some instances they did not confer resistance to imatinib. An increase in the frequency of T315I was not observed. The spectrum of mutations that emerged following therapy with second TKIs was compared to those that developed in previously published in vitro mutagenesis models. The mutations that persisted or developed following switch to new TKI were at sites also found in prior in vitro TKI mutagenesis assays.

Mutation regression/acquisition/persistence generally reflected the in vitro differential sensitivity predicted for each TKI. The mutations emerging after nilotinib and dasatinib also correlate to some extent with those that had the least in vitro sensitivity to these agents.

Preliminary analysis from phase 2 studies suggested that levels of response to new TKIs depend on the type of Bcr-Abl kinase domain mutation. Specifically, patients harboring native $\mathrm{Bcr}-\mathrm{Abl}$ and patients with a sensitive mutation had comparable response rates, whereas patients with mutations that confer intermediate sensitivity in vitro exhibited lower rates of major cytogenetic response. The clinical response of BCR-ABL with particular mutations has been modeled by classifying each mutation based on the in vitro $\mathrm{IC}_{50}$ for each drug against kinase activity for Bcr-Abl with that mutation into high-, intermediate- and low-sensitivity groups. Mutation sensitivity was defined by the $\mathrm{IC}_{50}$ value: $\mathrm{IC}_{50}$ values were $<3,<60$ and $>60 \mathrm{nM}$ for dasatinib, and $<50,<500$ and $>500 \mathrm{nM}$ for nilotinib (O'Hare et al 2007a) (Table 4). Investigating whether in vitro sensitivity of kinase domain mutation correlates with outcome of patients receiving second generation TKI, it was found that the outcome depends on the type of mutation, with mutations with predicted intermediate levels of sensitivity having decreased probability of response and event free survival, particularly in $\mathrm{CP}$. The correlation with $\mathrm{IC}_{50}$ was less evident in advanced phases suggesting more complex mechanisms of resistance. In the same line, overall survival of patients with T315I mutations was mostly found to be 
Table 4 Sensitivity of Bcr-Abl mutants to nilotinib ${ }^{56}$

Sensitive M244V, G250E, F3 I I L, F3 I 7L, M3 I 5T, L387M, H396P, H396R

Intermediate Q252H, Y253F, E255K, E255V,T3I5A, F359V, V379I

Resistant T3I5E

dependent on the stage of the disease (Jabbour et al 2007a). If this preliminary observation holds, rational decisions on drug and dose may require consideration of mutation type. In patients failing a TKI in whom a mutation with intermediate in vitro sensitivity to this agent is detected, change to an agent with better in vitro potency against such mutation may improve the response (Jabbour et al 2007b). The setting for outgrowth of different mutations and their subsequent response to different TKIs varies significantly. For example, mutations in codon 317, which impairs dasatinib binding, have been generated during in vitro mutagenesis with dasatinib but not nilotinib, and the F317L, in particular, has been reported following treatment with dasatinib. TKIs showing differential in vitro activity against this mutation, like nilotinib, may represent the best option for F317L-mutated tumors (Jabbour et al 2007c).

\section{Combination therapy}

There is growing interest in testing the hypothesis that administration of multiple Abl kinase inhibitors in early-phase patients, could be used to delay or prevent the emergence of drug-resistant clones (Shah et al 2005). The combination of 2 agents targeting different pathways involved in CML may significantly improve response rates and potentially increase survival.

Support for this concept is provided by preliminary preclinical investigations of the imatinib-nilotinib combination (Weisberg et al 2007). Additive/synergistic toxicity against both imatinib-sensitive and imatinib-resistant Bcr-Abl-expressing cells has been reported following coadministration of nilotinib and imatinib, in vitro and in vivo (Griffin and Weisberg 2005; Weisberg et al 2005; Weisberg et al 2007).

This cooperative activity could result from pharmacodynamic interactions with cell transporters. Preliminary data suggest that synergy between imatinib and nilotinib may occur at the level of the CML stem cell due to the ability of both imatinib and nilotinib to inhibit or act as substrates of the multidrug efflux transporter Abcg2, which confers resistance toward several anticancer drugs (Brendel et al 2007). It is also reported that imatinib and nilotinib might be taken up in cells by different mechanisms, with the influx, intracellular concentrations of imatinib, and consequently patient sensitivity to imatinib depending upon the organic cation transporter Oct-1, whereas nilotinib transport appears to be independent of Oct-1 (White et al 2006).

Both nilotinib and dasatinib efficiently block Bcr-Abl tyrosine kinase catalytic activity by binding to distinct, partially overlapping sites in the kinase domain. Cross resistance with dasatinib is limited to T315I, which is also the only mutant isolated at drug concentrations equivalent to maximal achievable plasma trough levels (O'Hare et al 2005). With drug combinations maximal suppression of resistant clone outgrowth was achieved at lower concentrations compared with single agents, suggesting that such combinations may be equipotent to higher dose single agents. A combination of low doses of dasatinib and low doses of nilotinib may effectively suppress the emergence of mutations other than T315I with an acceptable safety profile (Talpaz et al 2006; Jabbour et al 2007d). This approach needs to be eventually extended to include specific inhibitors of T315I Bcr-Abl kinase domain mutations. Alternatively, it is also important to explore the potential for synergy between nilotinib and other classes of inhibitors that work through mechanisms not involving inhibition of Abl tyrosine kinase activity (Fiskus et al 2006; O'Hare et al 2007b; O'Hare et al 2008).

\section{Conclusion}

The development of imatinib and subsequent studies into mechanisms of imatinib resistance have intensified the development of additional treatments for CML. Targeted agents have significantly improved the prognosis in CML. Further optimization of treatment responses may require patient-specific therapy based on an evaluation of disease characteristics.

\section{Disclosures}

EJ is on the speakers bureau of Novartis and Bristol-MyersSquibb. HK and JC have received grants from Novartis and Bristol-Myers-Squibb.

\section{References}

Aichberger KJ, Mayerhofer M, Krauth MT, et al. 2005. Low-level expression of proapoptotic Bcl-2-interacting mediator in leukemic cells in patients with chronic myeloid leukemia: role of $\mathrm{BCR} / \mathrm{ABL}$, characterization of underlying signaling pathways, and reexpression by novel pharmacologic compounds. Cancer Res, 65:9436-44.

Azam M, Latek RR, Daley GQ. 2003. Mechanisms of autoinhibition and STI-571/imatinib resistance revealed by mutagenesis of BCR-ABL. Cell, 112:831-43.

Barnes DJ, Melo JV. 2002. Cytogenetic and molecular genetic aspects of chronic myeloid leukaemia. Acta Haematol, 108:180-202. 
Bhatia R, Holtz M, Niu N, et al. 2003. Persistence of malignant hematopoietic progenitors in chronic myelogenous leukemia patients in complete cytogenetic remission following imatinib mesylate treatment. Blood, 101:4701-7.

Bradeen HA, Eide CA, O'Hare T, et al. 2006. Comparison of imatinib mesylate, dasatinib (BMS-354825), and nilotinib (AMN107) in an N-ethyl-N-nitrosourea (ENU)-based mutagenesis screen: high efficacy of drug combinations. Blood, 108:2332-8.

Brendel C, Scharenberg C, Dohse M, et al. 2007. Imatinib mesylate and nilotinib (AMN107) exhibit high-affinity interaction with ABCG2 on primitive hematopoietic stem cells. Leukemia, 21:1267-75.

Buchdunger E, Cioffi CL, Law N, et al. 2000. Abl protein-tyrosine kinase inhibitor STI571 inhibits in vitro signal transduction mediated by c-kit and platelet-derived growth factor receptors. J Pharmacol Exp Ther, 295:139-45.

Chan LC, Karhi KK, Rayter SI, et al. 1987. A novel abl protein expressed in Philadelphia chromosome positive acute lymphoblastic leukaemia. Nature, 325:635-7.

Corbin AS, Buchdunger E, Pascal F, et al. 2002. Analysis of the structural basis of specificity of inhibition of the Abl kinase by STI571. J Biol Chem, 277:32214-9.

Cortes J, Jabbour E, Kantarjian H, et al. 2007. Dynamics of BCR-ABL kinase domain mutations in chronic myeloid leukemia after sequential treatment with multiple tyrosine kinase inhibitors. Blood, 110:4005-11.

Cortes JE, O'Brien SM, Ferrajoli A, et al. 2008. 50. Efficacy of nilotinib (AMN107) in patients (Pts) with newly diagnosed, previously untreated philadelphia chromosome $(\mathrm{Ph})$ - positive chronic myelogenous leukemia in early chronic phase (CML-CP). J Clin Oncol, 26(May 20 Suppl): Abstr 7016 .

Dai Y, Rahmani M, Corey SJ, et al. 2004. A Bcr/Abl-independent, Lyn-dependent form of imatinib mesylate (STI-571) resistance is associated with altered expression of Bcl-2. J Biol Chem, 279:34227-39.

Deininger M, Buchdunger E, Druker BJ. 2005. The development of imatinib as a therapeutic agent for chronic myeloid leukemia. Blood, 105:2640-53.

Donato NJ, Wu JY, Stapley J, et al. 2003. BCR-ABL independence and LYN kinase overexpression in chronic myelogenous leukemia cells selected for resistance to STI571. Blood, 101:690-8.

Druker BJ, Guilhot F, O'Brien SG, et al. 2006. Five-year follow-up of patients receiving imatinib for chronic myeloid leukemia. N Engl J Med, 355:2408-17.

Faderl S, Talpaz M, Estrov Z, et al. 1999. Chronic myelogenous leukemia: biology and therapy. Ann Intern Med, 131:207-19.

Fiskus W, Pranpat M, Bali P, et al. 2006. Combined effects of novel tyrosine kinase inhibitor AMN107 and histone deacetylase inhibitor LBH589 against Bcr-Abl-expressing human leukemia cells. Blood, 108:645-52.

Giles FJ, Larson RA, Kantarjian HM, et al. 2008. Nilotinib in patients with Philadelphia chromosome-positive chronic myelogenous leukemia in blast crisis (CML-BC) who are resistant or intolerant to imatinib. J Clin Oncol, 26(May 20 Suppl):Abstr 7017.

Goldman JM, Hughes T, Radich J, et al. 2005. Continuing reduction in level of residual disease after 4 years in patients with CML in chronic phase responding to first-line imatinib (IM) in the IRIS Study. Blood, 106:Abstract 163.

Goldman JM, Melo JV. 2003. Chronic myeloid leukemia - advances in biology and new approaches to treatment. $N$ Engl J Med, 349:1451-64.

Golemovic M, Verstovsek S, Giles F, et al. 2005. AMN107, a novel aminopyrimidine inhibitor of Bcr-Abl, has in vitro activity against imatinib-resistant chronic myeloid leukemia. Clin Cancer Res, 11:4941-7.

Griffin JD, Weisberg EL. 2005. Simultaneous administration of AMN107 and imatinib in the treatment of imatinib-sensitive and imatinib-resistant chronic myeloid leukemia. Blood, (ASH Annual Meeting Abstracts), 106:694.

Hochhaus A, Hughes T. 2004. Clinical resistance to imatinib: mechanisms and implications. Hematol Oncol Clin North Am, 18:641-56, ix.
Hochhaus A, Kreil S, Corbin AS, et al. 2002. Molecular and chromosomal mechanisms of resistance to imatinib (STI571) therapy. Leukemia, 16:2190-6.

Hu Y, Liu Y, Pelletier S, et al. 2004. Requirement of Src kinases Lyn, Hck and Fgr for BCR-ABL1-induced B-lymphoblastic leukemia but not chronic myeloid leukemia. Nat Genet, 36:453-61.

Hughes T, Deininger M, Hochhaus A, et al. 2006. Monitoring CML patients responding to treatment with tyrosine kinase inhibitors: review and recommendations for harmonizing current methodology for detecting BCR-ABL transcripts and kinase domain mutations and for expressing results. Blood, 108:28-37.

Jabbour E, Cortes J, Kantarjian H. 2007. Dasatinib for the treatment of Philadelphia chromosome-positive leukaemias. Expert Opin Investig Drugs, 16:679-7. Erratum in: Expert Opin Investig Drugs, 2007; $16: 1135$.

Jabbour E, Hochhaus A, le Coutre P, et al. 2008. Minimal cross-intolerance between nilotinib and imatinib in patients with imatinib-intolerant chronic myelogenous leukemia (CML) in chronic phase (CP) or accelerated phase (AP). J Clin Oncol, 26(May 20 Suppl):Abstr 7063.

Jabbour E, Kantarjian H, Jones D, et al. 2006. Frequency and clinical significance of BCR-ABL mutations in patients with chronic myeloid leukemia treated with imatinib mesylate. Leukemia, 20:1767-73.

Jabbour E, Kantarjian H, Jones D, et al. 2007. Event-free survival in patients (pts) with chronic myeloid leukemia (CML) treated with 2nd generation tyrosine kinase inhibitors (TKI) after imatinib failure is dependent on the in vitro sensitivity of BCR-ABL kinase domain (KD) mutations. Blood, (ASH Annual Meeting Abstracts) 110:1941

Jabbour E, Kantarjian H, Jones D, et al. 2007. In vivo response to sequential tyrosine kinase inhibitors (TKI) in chronic myeloid leukemia (CML) modeled based on in vitro properties of particular BCR-ABL kinase domain (KD) mutations. Blood, (ASH Annual Meeting Abstracts) 110:1947.

Jabbour E, Kantarjian H, Jones D, et al. 2008. Clinical characteristics and outcome of patients with F317L BCR-ABL kinase domain mutation after therapy with tyrosine kinase inhibitors. Blood, Sep 25. [Epub ahead of print].

Jorgensen HG, Allan EK, Jordanides NE, et al. 2007. Nilotinib exerts equipotent antiproliferative effects to imatinib and does not induce apoptosis in CD34+ CML cells. Blood, 109:4016-9.

Kantarjian H, Giles F, Wunderle L, et al. 2006. Nilotinib in imatinibresistant CML and Philadelphia chromosome-positive ALL. $N$ Engl $J$ Med, 354:2542-51.

Kantarjian HM, Giles FJ, A. Hochhaus, et al. 2008. Nilotinib in patients with imatinib-resistant or -intolerant chronic myelogenous leukemia in chronic phase (CML-CP): Updated phase II results. J Clin Oncol, 26(May 20 Suppl):Abstr 7010.

Kantarjian HM, O'Brien S, Cortes J, et al. 2003. Imatinib mesylate therapy improves survival in patients with newly diagnosed Philadelphia chromosome-positive chronic myelogenous leukemia in the chronic phase: comparison with historic data. Cancer, 98:2636-42.

Koptyra M, Falinski R, Nowicki MO, et al. 2006. BCR/ABL kinase induces self-mutagenesis via reactive oxygen species to encode imatinib resistance. Blood, 108:319-27.

le Coutre P, Giles FJ, Apperley J, et al. 2008. Nilotinib in accelerated phase chronic myelogenous leukemia (CML-AP) patients with imatinibresistance or -intolerance: Update of a phase II study. J Clin Oncol, 26(May 20 Suppl):Abstr 7050.

Lionberger JM, Wilson MB, Smithgall TE. 2000. Transformation of myeloid leukemia cells to cytokine independence by Bcr-Abl is suppressed by kinase-defective Hck. J Biol Chem, 275:18581-5.

Litzow MR. 2006. Imatinib resistance: obstacles and opportunities. Arch Pathol Lab Med, 130:669-79.

Mahon F-X, Lagarde V, Manley PW, et al. 2004. Generation of resistance cell lines to AMN107, a new inhibitor of BCR-ABL and its effects on cell lines sensitive and resistant to imatinib. Blood, (ASH Annual Meeting Abstracts) 104:4670. 
Manley PW, Cowan-Jacob SW, Fendrich G, et al. 2005. Molecular interactions between the highly selective pan-Bcr-Abl inhibitor, AMN107, and the tyrosine kinase domain of Abl. Blood, (ASH Annual Meeting Abstracts) 106:3365.

Nagar B, Bornmann WG, Pellicena P, et al. 2002. Crystal structures of the kinase domain of c-Abl in complex with the small molecule inhibitors PD173955 and imatinib (STI-571). Cancer Res, 62:4236-43.

Nicolini FE, Corm S, Le QH, et al. 2006. Mutation status and clinical outcome of 89 imatinib mesylate-resistant chronic myelogenous leukemia patients: a retrospective analysis from the French intergroup of CML (Fi(phi)-LMC GROUP). Leukemia, 20:1061-6.

Nilotinib (Tasigna) for CML. 2008. Med Lett Drugs Ther, 50:26-7.

O'Brien SG, Guilhot F, Larson RA, et al. 2003. Imatinib compared with interferon and low-dose cytarabine for newly diagnosed chronic-phase chronic myeloid leukemia. N Engl J Med, 348:994-1004.

O'Hare T, Eide CA, Deininger MWN. 2007. Bcr-Abl kinase domain mutations, drug resistance, and the road to a cure for chronic myeloid leukemia. Blood, 110:2242-9.

O'Hare T, Eide CA, Tyner JW, et al. 2007. SGX70393 Inhibits Bcr-AblT315I in vitro and in vivo and completely suppresses resistance when combined with nilotinib or dasatinib. Blood, (ASH Annual Meeting Abstracts) 110:535.

O'Hare T, Eide CA, Tyner JW, et al. 2008. SGX393 inhibits the CML mutant Bcr-AblT315I and preempts in vitro resistance when combined with nilotinib or dasatinib. Proc Natl Acad Sci U S A, 105:5507-12.

O'Hare T, Walters DK, Stoffregen EP, et al. 2005. In vitro activity of Bcr-Abl inhibitors AMN107 and BMS-354825 against clinically relevant imatinib-resistant Abl kinase domain mutants. Cancer Res, 65:4500-5.

Ottmann OG, Larson RA, Kantarjian HM, et al. 2007. Nilotinib in patients (pts) with relapsed/refractory Philadelphia chromosome-positive acute lymphoblastic leukemia ( $\mathrm{Ph}+\mathrm{ALL})$ who are resistant or intolerant to imatinib. Blood, (ASH Annual Meeting Abstracts) 110:2815.

Picard S, Titier K, Etienne G, et al. 2007. Trough imatinib plasma levels are associated with both cytogenetic and molecular responses to standard-dose imatinib in chronic myeloid leukemia. Blood, 109:3496-9.

Quintas-Cardama A, Cortes JE. 2006. Chronic myeloid leukemia: diagnosis and treatment. Mayo Clin Proc, 81:973-88.

Ray A, Cowan-Jacob S, Manley PW, et al. 2007. Identification of Bcr/Abl point mutations conferring resistance to the Abl kinase inhibitor AMN107 by a random mutagenesis study. Blood, 109:5011-5.

Ries LAG MD, Krapcho M, et al. 2004. Sourveillance Epidemiology and End Results (SEER) Cancer Statistics Review, 1975-2004, National Cancer Institute, Bethesda, MD, USA. Accessed September 2007. URL: http://seercancergov/csr/1975_2004

Rowley JD. 1973. A new consistent chromosomal abnormality in chronic myelogenous leukaemia identified by quinacrine fluorescence and Giemsa staining [letter]. Nature, 243:290-3.
Roy L, Guilhot J, Krahnke T, et al. 2006. Survival advantage from imatinib compared with the combination interferon-alpha plus cytarabine in chronic-phase chronic myelogenous leukemia: historical comparison between two phase 3 trials. Blood, 108:1478-84.

Schindler T, Bornmann W, Pellicena P, et al. 2000. Structural mechanism for STI-571 inhibition of abelson tyrosine kinase. Science, 289:1938-42.

Shah NP, Nicoll JM, Branford S, et al. 2005. Molecular analysis of dasatinib resistance mechanisms in CML patients identifies novel BCR-ABL mutations predicted to retain sensitivity to imatinib: rationale for combination tyrosine kinase inhibitor therapy. Blood, (ASH Annual Meeting Abstracts) 106:1093.

Shah NP, Nicoll JM, Nagar B, et al. 2002. Multiple BCR-ABL kinase domain mutations confer polyclonal resistance to the tyrosine kinase inhibitor imatinib (STI571) in chronic phase and blast crisis chronic myeloid leukemia. Cancer Cell, 2:117-25.

Shah NP. 2005. Loss of response to imatinib: mechanisms and management. Hematology/the Education Program of the American Society of Hematology. American Society of Hematology. pp 183-7.

Talpaz M, Shah NP, Kantarjian H, et al. 2006. Dasatinib in imatinib-resistant Philadelphia chromosome-positive leukemias. $N$ Engl $\mathrm{J}$ Med, 354:2531-41.

Thomas J, Wang L, Clark RE, et al. 2004. Active transport of imatinib into and out of cells: implications for drug resistance. Blood, 104:3739-45.

Verstovsek S, Golemovic M, Kantarjian H, et al. 2005. AMN107, a novel aminopyrimidine inhibitor of p190 Bcr-Abl activation and of in vitro proliferation of Philadelphia-positive acute lymphoblastic leukemia cells. Cancer, 104:1230-6.

von Bubnoff N, Manley PW, Mestan J, et al. 2006. Bcr-Abl resistance screening predicts a limited spectrum of point mutations to be associated with clinical resistance to the Abl kinase inhibitor nilotinib (AMN107). Blood, 108:1328-33.

von Bubnoff N, Schneller F, Peschel C, et al. 2002. BCR-ABL gene mutations in relation to clinical resistance of Philadelphiachromosome-positive leukaemia to STI571: a prospective study. Lancet, 359:487-91.

Weisberg E, Catley L, Wright RD, et al. 2007. Beneficial effects of combining nilotinib and imatinib in preclinical models of BCR-ABL+ leukemias. Blood, 109:2112-20.

Weisberg E, Manley PW, Breitenstein W, et al. 2005. Characterization of AMN107, a selective inhibitor of native and mutant Bcr-Abl. Cancer Cell, 7:129-41.

Wendel HG, de Stanchina E, Cepero E, et al. 2006. Loss of p53 impedes the antileukemic response to BCR-ABL inhibition. Proc Natl Acad Sci U S A, 103:7444-9.

White DL, Saunders VA, Dang P, et al. 2006. OCT-1-mediated influx is a key determinant of the intracellular uptake of imatinib but not nilotinib (AMN107): reduced OCT-1 activity is the cause of low in vitro sensitivity to imatinib. Blood, 108:697-704. 
